\title{
PROPOSTA PARA A ESTIMATIVA DA ACURÁCIA DE REDES GEODÉSICAS HORIZONTAIS INTEGRANDO ANÁLISE DE ROBUSTEZ E DE COVARIÂNCIA
}

\author{
An approach for estimating the accuracy of horizontal geodetic networks based on \\ robustness and covariance analysis \\ IVANDRO KLEIN ${ }^{1,2}$ \\ MARCELO TOMIO MATSUOKA ${ }^{1,2}$ \\ JOÃO FRANCISCO GALERA MONICO ${ }^{3}$ \\ ${ }^{1}$ Laboratório de Pesquisas em Geodésia (LAGEO)/Departamento de \\ Geodésia/Instituto de Geociências - UFRGS \\ ${ }^{2}$ Programa de Pós-Graduação em Sensoriamento Remoto - UFRGS \\ Av. Bento Gonçalves, 9500, CEP 91501-970, \\ Cx. Postal 15001, Porto Alegre, RS, Brasil \\ ${ }^{3}$ Departamento de Cartografia - Faculdade de Ciências e Tecnologia/ \\ Universidade Estadual Paulista - FCT/Unesp \\ ivandroklein@gmail.com; tomiomatsuoka@gmail.com; galera@fct.unesp.br
}

\begin{abstract}
RESUMO
O objetivo deste trabalho é apresentar uma proposta para o controle de qualidade de redes geodésicas bidimensionais por meio das análises de robustez e de covariância. $\mathrm{Na}$ metodologia proposta, a acurácia (exatidão) posicional de cada ponto é estimada por meio de uma possível tendência posicional (via análise de robustez), além da própria precisão (incerteza) posicional (via análise de covariância), sendo ainda uma medida independente do datum adotado. Além da apresentação do desenvolvimento teórico do método, a aplicação do mesmo é demonstrada em um exemplo numérico. Os resultados obtidos indicam que, em geral, quanto maior o afastamento de um vértice desconhecido do(s) ponto(s) de controle da rede, maior é a propagação de erros aleatórios sobre este, e, quanto menos observações redundantes ao redor de um ponto, maior é a influência de possíveis erros (não aleatórios) sobre este, quando não detectados.

Palavras-chave: Análise de Robustez; Redes Geodésicas; Acurácia Posicional.
\end{abstract}




\section{ABSTRACT}

The goal of this paper is to present a methodology for quality control of horizontal geodetic networks through robustness and covariance analysis. In the proposed methodology, the positional accuracy of each point is estimated by a possible bias in their position (based on robustness analysis), in addition to its own positional precision (uncertainty) (through covariance analysis), being a measure independently from the choice of the datum. Besides presenting the theoretical development of the method, its application is demonstrated in a numerical example. The results indicate that, in general, the greater the distance of an unknown point to the control(s) point(s) of the network, the greater is the propagation of random errors on this unknown point, and the smaller the number of redundant observations around a unknown point, the greater the influence of possible (undetected) nonrandom errors on this point.

Keywords: Robustness Analysis; Geodetic Networks; Positional Accuracy.

\section{INTRODUCÃO}

A análise de tensões (strain analysis) é uma abordagem puramente geométrica para analisar a deformação de um corpo físico, baseada na teoria de elasticidade da mecânica, sendo aplicada para descrever a deformação (relativa) de um corpo com respeito a algum estado inicial (VANÍČEK et al., 1991).

A teoria de elasticidade e a análise de tensões são largamente utilizadas, por exemplo, nos campos da Matemática, da Construção Civil e da Engenharia Mecânica (ver, por exemplo, LOVE, 1944; HETNARSKI e IGNACZAK, 2010). A análise de tensões também já foi aplicada em Geofísica e Geodinâmica, como por exemplo, em estudos relativos a movimentos crustais e terremotos (ver, por exemplo, TERADA e MIYABE, 1929; TSUBOI, 1932; KASAHARA, 1958; POPE, 1966; SCHNEIDER, 1982).

Em Geodésia, o potencial de deformação de uma rede geodésica por meio da análise de tensões também foi investigado, podendo-se citar os trabalhos pioneiros de Thapa (1980), Vaníček et al. (1981), e Dare (1983), dentre outros.

Salienta-se que dentre os diferentes tipos de tensões, em redes geodésicas, considera-se deformações heterogêneas (isto é, diferentes para diferentes pontos/regiões da rede), e infinitesimais, pois, em geral, as deformações (por exemplo, da ordem de $\mathrm{cm}$ ) são muito menores do que as dimensões da rede (por exemplo, da ordem de $\mathrm{km}$ ). Além disso, a análise de tensões independe das propriedades físicas do material, e, na prática, o que se obtém é um campo discreto de deslocamento (por exemplo, somente para os pontos/nós da rede) como aproximação do (real) campo contínuo de deslocamento (VANÍČEK et al., 1991).

A existência de erros (não aleatórios) nas medidas realizadas em uma rede, também pode conduzir a "deformações" nesta rede. Baarda (1968) apresenta um procedimento estatístico de controle de qualidade, denominado "método $\beta$ " $(\beta$ method), que faz uso do chamado teste global ("global test") do ajustamento para a 
detecção de erros nas observações e/ou no modelo matemático, e do procedimento de teste data snooping para a identificação (localização) das observações suspeitas de estarem contaminadas por erros (não aleatórios).

No mesmo trabalho, Baarda propõe também a teoria de confiabilidade (reliability theory), que faz uso da confiabilidade interna (internal reliability) para estimar o menor erro detectável em uma observação (MDB - minimal detectable bias), seguindo esta metodologia de testes, e da confiabilidade externa (external reliability), que quantifica a influência deste erro (quando "não detectado") sobre cada um dos parâmetros incógnitos (estimados) do modelo (no caso, sobre as coordenadas dos vértices desconhecidos da rede geodésica).

Ressalta-se que tanto a análise de tensões quanto a teoria de confiabilidade consideram um deslocamento "virtual" (ou seja, nos valores numéricos das coordenadas), e não necessariamente, um deslocamento "real" ou "físico" dos vértices materializados no terreno.

Dentro deste contexto, a análise de robustez (robustness analysis) foi inicialmente concebida e formulada por meio do esforço conjunto de pesquisadores das Universidades de New Brunswick e Calgary no Canadá. Considera-se que a teoria de confiabilidade proposta por Baarda (1968) possui uma sólida fundamentação estatística, mas carece do tratamento da geometria do potencial de deformação de uma rede geodésica. Como a análise de tensões trata de forma rigorosa o potencial de deformação de uma rede geodésica, mas suas fundamentações estatísticas são relativamente fracas, foi proposto combinar as vantagens desta abordagem com as da teoria de confiabilidade em uma única técnica denominada "análise de robustez" (VANÍČEK et al., 1991). O termo "robustez" foi escolhido para refletir a terminologia estatística contemporânea, pois estimadores que são "insensíveis" a presença de erros grosseiros (ou outliers) nas observações são chamados de estimadores "robustos" (VANÍČEK et al., 2001).

A análise de robustez vem sendo largamente estudada pelos pesquisadores das Universidades de New Brunswick e Calgary deste então, e dentre os resultados publicados, pode-se citar Ong (1993), Vaníček et al. (1996), e Vaníček et al. (2001), com as devidas correções para este último em Craymer e Vaníček (2002).

A análise de robustez também foi investigada por outros pesquisadores, como por exemplo, nos trabalhos de Seemkooei (2001a, 2001b), que analisou a relação entre os números de redundância das observações (reliability numbers) e os parâmetros de robustez, para fins de otimização de redes geodésicas, e em Gambin et al. (2008), onde são feitas analogias entre a análise de robustez de redes geodésicas e a teoria de tensões (strain theory) utilizada na mecânica contínua (continuum mechanics). Em Hsu e Li (2004), é apresentada a decomposição das primitivas de deformação (ou parâmetros de robustez) em duas componentes, uma de caráter "local" (relativa à redundância da própria observação), e outra complementar a esta, bem como a aplicação em uma rede geodésica. Em Hsu et al. (2008), é apresentada uma demonstração matemática para o fato de redes geodésicas tridimensionais apresentarem uma robustez superior em planimetria e inferior em 
altimetria. Em Marjetic et al. (2010), são investigadas as propriedades probabilísticas e estatísticas dos tensores de tensão e de rotação (strain and rotation tensors).

Outra contribuição relevante sobre o tema é a tese de Berber (2006), onde é discutida em detalhes a aplicação da análise de robustez em redes geodésicas unidimensionais, bidimensionais e tridimensionais, bem como propostos valores limiares (threshold values) para o controle de qualidade das redes geodésicas por meio desta técnica, além da investigação de medidas denominadas "invariantes de tensão" (strain invariants), cuja invariância diz respeito ao referencial (datum) adotado. Dentre os trabalhos resultantes das contribuições e conclusões desta pesquisa, pode-se citar ainda Berber et al. (2003), Berber et al. (2006), Vaníček et al. (2008), e Berber et al. (2009).

Finalmente, em âmbito nacional, poucos estudos relativos à análise de tensões e robustez em redes geodésicas são encontrados, podendo-se citar os trabalhos de Teixeira e Ferreira (2002), e de Cavalheri e Chaves (2012a, 2012b).

Dentro deste contexto, o objetivo deste trabalho é apresentar uma proposta para o controle de qualidade de redes geodésicas bidimensionais por meio das análises de robustez e de covariância. Na metodologia proposta, a acurácia (exatidão) posicional de cada ponto é estimada por meio de uma possível tendência em sua posição (via análise de robustez), além de sua própria precisão (incerteza) posicional (via análise de covariância), com a vantagem dos resultados obtidos serem independentes de fatores externos como o referencial adotado, sendo reflexos da própria geometria/configuração da rede geodésica e do tipo e qualidade (propriedades estatísticas) de suas observações.

Além da revisão teórica, o desenvolvimento da metodologia proposta é apresentado e aplicado em um exemplo numérico.

\section{ANÁLISE DE ROBUSTEZ DE REDES GEODÉSICAS}

Considerando que os possíveis deslocamentos dos pontos de uma rede geodésica sejam causados por erros (não detectados) nas observações, estes podem ser estimados por meio da confiabilidade externa, que na realidade é uma medida de confiabilidade interna, mas no espaço dos parâmetros ao invés de no espaço das observações (VANÍČEK et al., 2001). Entretanto, estas estimativas para os (possíveis) deslocamentos dos pontos por meio da confiabilidade externa das observações são datum dependentes, ou seja, dependem do referencial adotado.

Idealmente, a análise do potencial de deformação de uma rede geodésica deve ser dependente somente da própria geometria/configuração da rede geodésica e do tipo e precisão (propriedades estatísticas) de suas observações, devendo ser independente de fatores externos como o datum utilizado (definido por meio de injunções no ajustamento), para que diferentes geodesistas, utilizando diferentes data, cheguem aos mesmos resultados e conclusões (VANÍČEK et al., 1991).

É importante salientar que os deslocamentos de um ponto $\mathrm{P}_{\mathrm{i}}\left(\Delta \mathrm{x}_{\mathrm{i}}, \Delta \mathrm{y}_{\mathrm{i}}\right)$, estimados por meio da confiabilidade externa, dependem do referencial adotado 
(principalmente por causa da orientação dos eixos), mas a sua norma, ou seja, o deslocamento resultante $-\sqrt{\Delta x_{i}{ }^{2}+\Delta y_{i}^{2}}$, não depende do referencial adotado. Também ressalva-se que em Baarda (1977), é proposta outra medida de confiabilidade externa, denominada confiabilidade externa relativa (relative external reliability measure), que é datum independente (embora seja apenas um escalar para cada observação, para ser utilizado principalmente em análises relativas).

De qualquer forma, além desta questão de independência do datum, a análise de robustez, por se utilizar da análise de tensões, fornece uma descrição mais completa e detalhada do potencial de deformação da rede (ponto a ponto), por meio de três medidas independentes representando robustez em escala, em orientação e em configuração local (VANÍČEK et al., 2001). Portanto, a "robustez" de uma rede deve ser compreendida como a capacidade da rede em "resistir" a deformações causadas por erros "não detectados” nas observações (VANÍČEK et al., 1991).

Para melhor compreensão do conceito de deformação de uma rede geodésica, inicialmente, considere o caso de um ponto $\mathrm{P}_{\mathrm{i}}$ em um espaço bidimensional (como por exemplo, em um plano topográfico local), cujas coordenas neste plano são dadas por $\left(\mathrm{x}_{\mathrm{i}}, \mathrm{y}_{\mathrm{i}}\right)$. Considere agora que ocorram alterações (deslocamentos) nos valores das coordenadas deste ponto, e que estas sejam dadas, respectivamente, por $\Delta \mathrm{x}_{\mathrm{i}}$ e $\Delta \mathrm{y}_{\mathrm{i}}$.

Matematicamente, a relação entre os deslocamentos $\left(\Delta \mathrm{x}_{\mathrm{i}}, \Delta \mathrm{y}_{\mathrm{i}}\right)$ e a posição “inicial" $\left(\mathrm{x}_{\mathrm{i}}, \mathrm{y}_{\mathrm{i}}\right)$ do ponto $\mathrm{P}_{\mathrm{i}}$ em questão é dada por:

$$
\Delta x_{i}=\frac{d \Delta x}{d x} x_{i}, \Delta y_{i}=\frac{d \Delta y}{d y} y_{i}
$$

onde $\frac{d \Delta x}{d x}$ e $\frac{d \Delta y}{d y}$ são as taxas de deslocamento (variação) ao longo dos eixos x e y, respectivamente (considerando o caso simplificado em que os deslocamentos entre os dois eixos sejam independentes). Uma analogia pode ser feita entre a equação (1) e o movimento retilíneo uniforme (MRU), onde o deslocamento total de um objeto ao longo de um eixo é igual ao produto entre a taxa de deslocamento deste objeto em relação ao tempo (velocidade), e o tempo total de deslocamento do referido objeto. Considerando agora o caso de dois pontos $\left(\mathrm{P}_{\mathrm{i}}\right.$ e $\left.\mathrm{P}_{\mathrm{j}}\right)$ em um espaço bidimensional, cujas coordenadas são dadas, respectivamente, por $\left(x_{i}, y_{i}\right)$ e $\left(x_{j}, y_{j}\right)$, pode-se relacionar, linearmente, os possíveis deslocamentos destes dois pontos $\left(\Delta \mathrm{x}_{\mathrm{i}}, \Delta \mathrm{y}_{\mathrm{i}}\right)$ e $\left(\Delta \mathrm{x}_{\mathrm{j}}, \Delta \mathrm{y}_{\mathrm{j}}\right)$ com as suas posições iniciais - $\left(\mathrm{x}_{\mathrm{i}}, \mathrm{y}_{\mathrm{i}}\right)$ e $\left(\mathrm{x}_{\mathrm{j}}, \mathrm{y}_{\mathrm{j}}\right)$, por meio da seguinte equação (GAMBIN et al., 2008): 


$$
\begin{aligned}
& \Delta x_{j}-\Delta x_{i}=\frac{\partial \Delta x_{i}}{\partial x}\left(x_{j}-x_{i}\right)+\frac{\partial \Delta x_{i}}{\partial y}\left(y_{j}-y_{i}\right) \\
& \Delta y_{j}-\Delta y_{i}=\frac{\partial \Delta y_{i}}{\partial x}\left(x_{j}-x_{i}\right)+\frac{\partial \Delta y_{i}}{\partial y}\left(y_{j}-y_{i}\right)
\end{aligned}
$$

onde $\frac{\partial \Delta x_{i}}{\partial x} \mathrm{e} \frac{\partial \Delta x_{i}}{\partial y}$ são as taxas de deslocamento (sobre o ponto $\mathrm{P}_{\mathrm{i}}$ ) ao longo da direção x em relação aos eixos x e y, respectivamente, e $\frac{\partial \Delta y_{i}}{\partial x} \mathrm{e} \frac{\partial \Delta y_{i}}{\partial y}$ são as taxas de deslocamento (sobre o ponto $\mathrm{P}_{\mathrm{i}}$ ) ao longo da direção y em relação aos eixos $\mathrm{x}$ e $\mathrm{y}$, respectivamente. A equação (2) define um campo de deslocamento (displacement field) sobre o plano em questão e ao redor do ponto $\mathrm{P}_{\mathrm{i}}$, utilizando o método de diferenças para modelos de deformação linear.

Como as derivadas parciais presentes na equação (2) são os gradientes de deslocamento sobre o ponto $\mathrm{P}_{\mathrm{i}}$ em relação aos eixos x e y, a matriz de deformação (ou de tensão) sobre o ponto $\mathrm{P}_{\mathrm{i}}$ (deformation or strain matrix), também conhecida como tensor gradiente com respeito a posição, é dada por (VANÍČEK et al., 1991):

$$
E_{i}=\nabla\left(\Delta x_{i}, \Delta y_{i}\right)=\left[\begin{array}{cc}
\frac{\partial \Delta x_{i}}{\partial x} & \frac{\partial \Delta x_{i}}{\partial y} \\
\frac{\partial \Delta y_{i}}{\partial x} & \frac{\partial \Delta y_{i}}{\partial y}
\end{array}\right]
$$

Na análise de robustez, para o cálculo dos elementos da matriz de deformação definida em (3) sobre cada ponto $\mathrm{P}_{\mathrm{i}}$, se utiliza da teoria de confiabilidade proposta inicialmente em Baarda (1968). Desta forma, a confiabilidade interna de uma observação diz respeito ao menor erro detectável desta observação (minimal detectable bias - MDB), para o procedimento de teste data snooping, com um dado nível de significância $(\alpha)$ e poder do teste $(\gamma)$. Para cada observação $\left(\mathrm{lb}_{\mathrm{i}}\right)$, o seu MDB $\left(\nabla l b_{i}\right)$, em módulo, é dado por (BAARDA, 1968; TEUNISSEN, 2006):

$$
\nabla l b_{i}=\sqrt{\frac{\lambda_{0}}{c_{l b(i)}^{T} P \Sigma_{V} P c_{l b(i)}}},
$$

onde na equação (4), $\lambda_{0}$ corresponde ao "parâmetro de não centralidade" do modelo (non-centrality parameter), obtido em função do nível de significância, graus de liberdade e poder do teste (ver, por exemplo, BAARDA, 1968; TEUNISSEN, 2006), P é a matriz peso (usualmente tomada como sendo o inverso da matriz de covariância das observações), $\Sigma_{\mathrm{V}}$ é a matriz de covariância dos resíduos ajustados, e 
$\mathrm{c}_{\mathrm{lb}(\mathrm{i})}$ é um vetor canônico unitário, contendo a unidade na linha referente a i-ésima observação testada e zeros nas demais, ou seja: $c_{l b(i)}^{T}=[00 \ldots 0100 \ldots 0]^{T}$.

A confiabilidade externa de uma observação $\left(\nabla X_{i}\right)$ é um vetor que quantifica a influência do menor erro detectável desta observação $\left(\nabla \mathrm{lb}_{\mathrm{i}}\right)$, também interpretado como "erro máximo não detectado", sobre cada um dos parâmetros incógnitos do ajustamento (no caso de redes geodésicas em geral, nas coordenadas dos pontos desconhecidos), sendo dada pela expressão (BAARDA, 1968):

$$
\nabla X_{i}=\left(A^{T} P A\right)^{-1} A^{T} P\left(c_{l b(i)} \nabla l b_{i}\right)
$$

onde na equação (5), $A$ corresponde a matriz design (ou matriz jacobiana) do ajustamento. Como para cada observação $\left(\mathrm{lb}_{\mathrm{i}}\right)$, se tem um MDB $\left(\nabla \mathrm{lb}_{\mathrm{i}}\right)$, ao todo, temse " $n$ " vetores de confiabilidade externa distintos (sendo "n" o número de observações ou medidas realizadas). Desta forma, considerando um possível erro (não detectado) em uma observação $l_{b}$, os deslocamentos na posição de dois pontos quaisquer da rede na equação (2) podem ser substituídos pelos respectivos valores de confiabilidade externa do vetor $\nabla X_{i}$, obtidos por meio da equação (5).

Portanto, considerando que para calcular o campo de deslocamento ao redor de um ponto qualquer $\mathrm{P}_{\mathrm{i}}$, serão utilizados outros " $\mathrm{k}$ " pontos da rede geodésica (podendo estes, por exemplo, serem os pontos que estão "interligados" com o ponto em questão por uma ou mais observações, ou ainda os pontos que estão situados até uma certa distância de $\mathrm{P}_{\mathrm{i}}$ ), a equação (2) pode ser expressa na forma matricial (GAMBIN et al., 2008):

$$
\Delta u=B e_{i}
$$

onde $\Delta \mathrm{u}$ é um vetor (de dimensão $2 \mathrm{k}$ por 1 ), contendo as diferenças de deslocamentos (valores de confiabilidade externa) nos eixos x e y entre o ponto $\mathrm{P}_{\mathrm{i}} \mathrm{e}$ cada um dos $\mathrm{k}$ pontos considerados, B é uma matriz (de dimensão $2 \mathrm{k}$ por 4), contendo zeros e/ou as diferenças de coordenadas entre o ponto $\mathrm{P}_{\mathrm{i}}$ e cada um dos $\mathrm{k}$ pontos considerados (dependendo se a equação considerada é relativa ao eixo x ou $\mathrm{y}$, similar ao caso das transformações isogonal e afim $2 \mathrm{D}$ ), e e é o vetor (de dimensão 4 por 1) contendo os gradientes de deslocamento (elementos da matriz de deformação em $\mathrm{P}_{\mathrm{i}}$ ), ou seja: $e_{i}^{T}=\left[\frac{\partial \Delta x_{i}}{\partial x} \frac{\partial \Delta x_{i}}{\partial y} \frac{\partial \Delta y_{i}}{\partial x} \frac{\partial \Delta y_{i}}{\partial y}\right]^{T}$. Eventualmente, o sistema de equações em (6) poderá apresentar singularidade, como no caso em que todos os pontos considerados sejam colineares, não definindo um plano, ou ainda, no caso em que a rede não possua observações redundantes para a determinação do ponto $\mathrm{P}_{\mathrm{i}}$, como por exemplo, por meio da intersecção de apenas duas distâncias no plano local (ONG, 1993). 
Como os deslocamentos dos pontos são estimados a priori por meio da confiabilidade externa da observação considerada (equação 5), e os valores das coordenadas dos pontos são estimados por meio do ajustamento por mínimos quadrados da rede, as únicas incógnitas presentes na equação (6) são os elementos da matriz de deformação $\mathrm{E}_{\mathrm{i}}$. Considerando $(\mathrm{k} \geq 3)$ pontos adjacentes ao ponto $\mathrm{P}_{\mathrm{i}}$, pode-se aplicar um ajustamento por mínimos quadrados para estimar os elementos da matriz de deformação (considerando um peso igual para todos os deslocamentos estimados por meio da confiabilidade externa), resultando em (GAMBIN et al., 2008):

$$
e_{i}=\left(B^{T} B\right)^{-1} B^{T} \Delta u
$$

Uma estratégia de ponderação para os elementos do vetor de deslocamentos $(\Delta u)$, introduzindo uma matriz peso na equação (7) em função das distâncias entre os diferentes pontos considerados, pode ser obtida em Marjetic et al. (2010).

Ressalta-se que existem várias maneiras de se calcular os elementos da matriz de tensão que definem o campo de deslocamento ao redor do ponto $\mathrm{P}_{\mathrm{i}}$, podendo, por exemplo, serem utilizados apenas os pontos "interligados" a este por meio de uma ou mais observações, pontos situados até uma certa distância de $\mathrm{P}_{\mathrm{i}}$, ou todos os vértices desconhecidos da rede. Além disso, ao invés do método de diferenças para modelos de deformação linear apresentado na equação (2), podem ser utilizados outros modelos matemáticos para aproximar o campo de deslocamento ao redor do ponto $\mathrm{P}_{\mathrm{i}}$, como por exemplo, uma superfície simples como um plano (matematicamente descrito por um polinômio de grau 1, cuja expressão é diferente da equação 2, ver, por exemplo, VANÍČEK et al., 2001; HSU e LI, 2004). Polinômios algébricos de ordem elevada, geralmente, não são adequados para aplicações da análise de robustez em redes geodésicas, pois eles tendem a produzir gradientes errôneos quando os pontos não estão regularmente (homogeneamente) distribuídos no espaço (VANíČEK et al., 1991).

Uma estratégia relativamente simples para a análise do ajustamento do campo de deformação ao redor de um ponto $\mathrm{P}_{\mathrm{i}}$, considerando o modelo matemático e o número de pontos utilizados, pode ser obtida em Gambin et al. (2008). Salienta-se também que, para cada ponto da rede, tem-se " $n$ " matrizes de deformação diferentes (cada uma relativa ao MDB, e consequentemente, a confiabilidade externa de uma dada observação $l_{\mathrm{i}}$ ).

Ainda sobre a determinação dos elementos da matriz de tensão, Vaníček et al. (1996) concluem que, limitando o cálculo da matriz de tensão de um ponto $P_{i}$ para observações que não estejam mais do que "3 níveis de ligação" do ponto de interesse, se obtêm praticamente os mesmos resultados que seriam obtidos caso todas as observações fossem consideradas, além de diminuir o custo computacional, especialmente para redes geodésicas com um número relativamente alto de observações. Além disso, a análise de tensões é uma técnica diferencial, e por isto, é 
muito importante a seleção adequada dos pontos que serão utilizados no cálculo do campo de deslocamento ao redor de um ponto $\mathrm{P}_{\mathrm{i}}$, pois pontos muito distantes, por exemplo, tendem a "suavizar" os resultados. Os mesmos autores testaram a triangulação de Delaunay para a seleção dos pontos utilizados no cálculo de $\mathrm{E}_{\mathrm{i}}$ para cada vértice, mas concluíram que o método mais adequado é de fato ponderar a influência dos $(\mathrm{k})$ pontos selecionados de acordo com a sua distância ao ponto de interesse $\mathrm{P}_{\mathrm{i}}$, como é proposto, por exemplo, em Marjetic et al. (2010).

A matriz de deformação em torno de um ponto $\mathrm{P}_{\mathrm{i}}$ pode ser decomposta, ainda, em uma parte simétrica, cujos elementos descrevem uma deformação diferencial simétrica (também denominada "Tensor de Cauchy"), e em uma parte antissimétrica, que descreve uma rotação diferencial no ponto de interesse $\mathrm{P}_{\mathrm{i}}$ (ver, por exemplo, VANíČEK et al., 2001). Entretanto, estes elementos da matriz de deformação não possuem uma interpretação geométrica muito simples, e os termos da parte simétrica dependem ainda da orientação do sistema de referência utilizado.

Visando obter medidas que independam do referencial (datum) utilizado, e que apresentem uma interpretação mais conveniente e ilustrativa, Vaníček et al. (1991) se utilizam de três primitivas ou medidas de tensão (strain primitives or measures), também denominadas primitivas ou medidas de deformação (deformation primitives or measures), sendo estas: a tensão ou dilatação média - mean strain or dilatation $(\sigma)$, a rotação diferencial média - average differential rotation $(\omega)$, e o cisalhamento total - total shear $(\gamma)$. Cada uma destas três primitivas de tensão $(\sigma, \omega, \gamma)$ pode ser obtida em função dos quatro elementos da matriz de deformação $\left(E_{i}\right)$ por meio das seguintes expressões (VANÍČEK et al., 1991):

$$
\begin{aligned}
& \sigma=\frac{1}{2} \operatorname{traço~}\left(E_{i}\right)=\frac{1}{2}\left(\frac{\partial \Delta x_{i}}{\partial x}+\frac{\partial \Delta y_{i}}{\partial y}\right) \\
& \omega=\frac{1}{2}\left(\frac{\partial \Delta y_{i}}{\partial x}-\frac{\partial \Delta x_{i}}{\partial y}\right) \\
& \gamma=\sqrt{\tau^{2}+v^{2}} ; \tau=\frac{1}{2}\left(\frac{\partial \Delta x_{i}}{\partial x}-\frac{\partial \Delta y_{i}}{\partial y}\right), v=\frac{1}{2}\left(\frac{\partial \Delta x_{i}}{\partial y}+\frac{\partial \Delta y_{i}}{\partial x}\right)
\end{aligned}
$$

onde na equação (8), $\tau$ corresponde ao cisalhamento puro (pure shear) e $v$ corresponde ao cisalhamento simples (simple shear). A rotação diferencial média $(\omega)$ pode ser decomposta, ainda, na rotação de "bloco" - $\omega_{0}$ (ou seja, uma rotação "comum" ou "média" para toda a rede), e na rotação diferencial local $-\delta \omega$, ou seja, no ponto propriamente dito (ver, por exemplo, VANíČEK et al., 2001). Sobre as unidades, $\sigma$ e $\gamma$ são grandezas adimensionais, enquanto $\omega$ é expressa em radianos.

Naturalmente, outras medidas de deformação em função dos quatro elementos da matriz de tensão $E_{i}$ poderiam ser consideradas, sendo que a vantagem de se utilizar as três apresentadas é a sua interpretação mais intuitiva (ONG, 1993): a dilatação média descreve uma contração ou expansão "média" ao redor de um 
ponto, podendo ser considerada como uma deformação em escala (causada, por exemplo, por medidas de distâncias), enquanto a rotação diferencial média pode ser considerada como uma deformação em orientação (causada, por exemplo, por alterações no azimute). Valores positivos de $\sigma$ indicam "expansão" na escala da rede e valores negativos para esta indicam "contração" na escala da rede ao redor de um ponto $\mathrm{P}_{\mathrm{i}}$, enquanto valores positivos de $\omega$ indicam uma rotação no sentido antihorário e valores negativos para esta indicam uma rotação no sentido horário.

O cisalhamento simples altera o ângulo entre duas linhas, enquanto o cisalhamento puro altera a separação entre duas linhas, e desta forma, o cisalhamento total (sempre positivo) pode ser interpretado como uma deformação na "configuração local" em torno de um ponto $\mathrm{P}_{\mathrm{i}}$, causada, por exemplo, por medidas de ângulos e direções entre este e os demais pontos da rede (para maiores detalhes, ver, por exemplo, DARE, 1983; VANÍČEK et al., 1991; e ONG, 1993).

A Figura 1 ilustra graficamente o efeito dos quatro elementos da matriz de tensão, bem como o cisalhamento puro (em 1a) e o cisalhamento simples (em 1b).

Figura 1- Ilustração geométrica dos quatro elementos da matriz de tensão $\mathrm{E}_{\mathrm{i}}$ e do: a) cisalhamento puro e b) cisalhamento simples (Fonte: Adaptado de Ong, 1993).

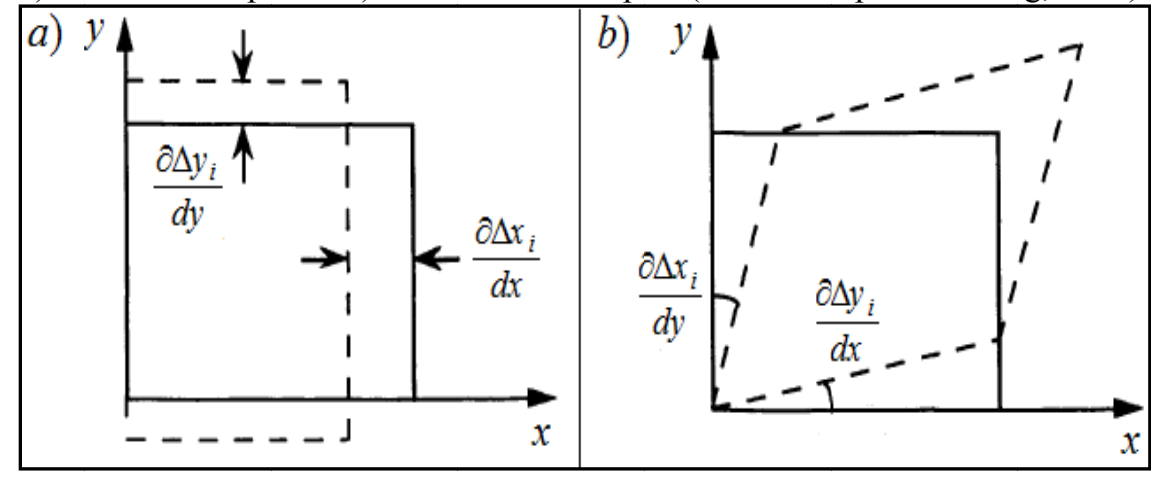

Para o cálculo da matriz de deformação em (3), são utilizados os valores da confiabilidade externa, que são datum dependentes. Entretanto, em Vaníček et al. (2001), demonstra-se matematicamente a independência das três medidas de deformação $(\sigma, \omega, \gamma)$ em relação a mudanças na origem (translação) e na orientação do referencial, enquanto alterações na escala resultam em efeitos de apenas segunda ordem. Ou seja, no plano, estas três primitivas de tensão podem ser consideradas "invariantes de tensão" (entretanto, o cisalhamento simples $-v$, e o cisalhamento puro $-\tau$, dependem da orientação do referencial). Para uma discussão mais detalhada sobre as primitivas de deformação e a independência do datum, incluindo os cenários unidimensionais (onde apenas uma medida de deformação é considerada - dilatação) e tridimensionais (onde o cisalhamento total é datum dependente), bem 
como a relação matemática entre os invariantes de tensão em duas e três dimensões, ver Berber (2006) e também Vaníček et al. (2008).

Salienta-se que estas discussões e conclusões são relativas aos casos em que o referencial (datum) é especificado por meio do uso de injunções mínimas (minimal constraints). Se o ajustamento contiver mais injunções do que o mínimo necessário, este será "sobre-injuncionado" (overconstrained), e a rede geodésica apresentará um potencial de deformação diferente do que o obtido utilizando injunções mínimas, devido ao uso de injunções (condições) em excesso. Esta afirmação é válida para o uso de injunções absolutas, mas não para o uso de injunções relativas ou ponderadas (weighted constraints), que pertencem de maneira intrínseca à rede (assim como as próprias observações), pois o fato de que as injunções relativas também podem fornecer um datum para o ajustamento, suprindo a deficiência de posto do mesmo, é apenas incidental (VANÍČEK et al., 1991).

Como para cada ponto da rede geodésica tem-se " $n$ " matrizes de tensão diferentes (cada uma relativa a uma observação), para cada ponto, tem-se ao todo “3n" primitivas de tensão (ou seja, dilatação média $-\sigma$, rotação diferencial média $\omega$ e cisalhamento total $-\gamma$ ). Além disso, quanto maior (em módulo) o valor de uma dada primitiva de tensão, "menos robusta" é a rede geodésica no ponto em questão, e, portanto, na prática, se está interessado somente nas deformações máximas em cada ponto (denotadas por $\sigma_{\text {máx }}, \omega_{\text {máx }}, \gamma_{\text {máx }}$ ). Estes valores máximos em cada ponto da rede (considerando todos os valores obtidos com as "n" observações) são denominados parâmetros de robustez (robustness parameters), sendo: $\sigma_{\text {máx }}$ robustez em escala (robustness in scale), $\omega_{\text {máx }}$ - robustez em orientação (robustness in orientation) e $\gamma_{\text {máx }}$ - robustez em configuração local (robustness in local configuration). Além disso, considerando que uma rede geodésica é tão "robusta" quanto o seu "elo mais fraco" ("credo of the weakest link", ver, DARE, 1983), para descrever a robustez de uma rede geodésica, pode-se utilizar somente dos valores máximos (em módulo) para cada uma destas três primitivas de tensão, obtidos considerando todas as observações e todos os pontos da rede (ver, por exemplo, SEEMKOOEI, 2001a, 2001b).

Desta forma, as possíveis distorções existentes na rede geodésica podem ser expressas tanto em termos da confiabilidade externa, ou seja, do "ponto de vista das observações", quanto em termos dos parâmetros de robustez, ou seja, do "ponto de vista dos pontos da rede" (SEEMKOOEI, 2001b). É importante ressaltar que na análise de tensões, pode-se utilizar para o cálculo das primitivas de deformação, ao invés do MDB de uma observação, o seu respectivo desvio-padrão, ou seja, verificar como os vértices da rede "respondem" a uma dada "incerteza" (erro aleatório) no valor de uma observação. De qualquer modo, recomenda-se que a análise de robustez seja utilizada de maneira conjunta com a análise de covariância, pois ambas fornecem descrições diferentes da rede, a primeira analisando as possíveis distorções causadas por erros grosseiros (não detectados), e a segunda analisando somente a propagação de erros de natureza aleatória (VANÍČEK et al., 1991). Resultados e discussões de estudos comparativos entre ambas as abordagens podem 
ser obtidos, por exemplo, em Vaníček et al. (1991), Ong (1993), e Vaníček et al. (2001).

Sobre os parâmetros de robustez, em Berber (2006) é apresentada uma "estratégia remedial" (remedial strategy), caso a rede geodésica apresente certa deficiência em robustez. Como os parâmetros de robustez dependem, essencialmente, da geometria/configuração da rede (ou seja, da redundância do problema), e da precisão das observações, geralmente, se adicionando observações (de precisão não inferior às demais), e/ou melhorando a qualidade das observações (por meio de métodos e/ou equipamentos mais precisos), espera-se uma redução nos valores dos parâmetros de robustez. Além disso, se a deficiência ocorrer em termos de escala (representada pela dilatação média), devem-se adicionar observações de distâncias à rede, enquanto que, se a deficiência ocorrer em termos de orientação (representada pela rotação diferencial média), devem-se adicionar observações de ângulos (ou azimutes) e distâncias à rede. Outras considerações pertinentes e estratégias para o aumento da robustez de uma rede geodésica podem ser obtidas em Seemkooei (2001a, 2001b), e Hsu e Li (2004).

Gambin et al. (2008) ressaltam que uma desvantagem dos parâmetros de robustez é que esta não apresenta uma ligação direta com a análise de covariância tradicional - covariance analysis (ver, por exemplo, VANÍČEK e KRAKIWSKY, 1986; GHIILANI e WOLF, 2006), e desta forma, embora as primitivas de deformação apresentem um significado intuitivo, para aplicações práticas, é difícil estabelecer valores limites para o controle de qualidade de redes geodésicas, considerando critérios tanto de precisão quanto de robustez. Os referidos autores também afirmam que uma das abordagens possíveis para contornar este problema seria o uso dos tradicionais conceitos de confiabilidade interna e externa, porém, cujo desenvolvimento matemático apresentasse valores datum independentes. Em Berber (2006), entretanto, este problema é contornado por meio de um procedimento de cálculo para os deslocamentos dos pontos em função das matrizes de tensões (conforme será visto na próxima seção).

Outra limitação na aplicação da análise de robustez em redes geodésicas é que estas são, intrinsecamente, de natureza bidimensional, pois as variações em altitude, em geral, são muito menores do que as extensões horizontais da rede sobre a superfície terrestre (VANÍČEK et al., 1991). Por não fazer parte do escopo deste trabalho, uma discussão detalhada sobre as limitações da análise de robustez em redes unidimensionais (como as redes de nivelamento geométrico e os circuitos gravimétricos, por exemplo), pode ser obtida em Berber (2006), enquanto aplicações e limitações desta técnica em redes tridimensionais (como redes GNSS e as fototriangulações, por exemplo), podem ser obtidas em Berber (2006), Hsu et al. (2008), Vaníček et al. (2008), e Berber et al. (2009).

Após esta revisão teórica sobre o tema e considerando as discussões expostas, na próxima seção, será apresentada uma proposta metodológica para o controle de qualidade de redes geodésicas horizontais, tendo como objetivo estimar a acurácia dos pontos da rede.

Bol. Ciênc. Geod., sec. Artigos, Curitiba, v. 19, no 4, p.525-547, out-dez, 2013. 


\section{METODOLOGIA PROPOSTA}

Em Vaníček et al. (1996), são feitas discussões e considerações a respeito da classificação de redes geodésicas de acordo com a sua precisão e robustez. Em Berber (2006), é proposta uma metodologia de controle de qualidade para redes geodésicas bidimensionais e tridimensionais por meio da análise de robustez, considerando as especificações dadas pela Divisão Canadense de Levantamentos Geodésicos. Neste trabalho, pretende-se apresentar uma metodologia que não atenda as especificações de um determinado país e/ou orgão, mas que possa ser empregada de uma forma geral (respeitando, evidentemente, as normas vigentes em cada país).

A metodologia aqui proposta para o controle de qualidade de redes geodésicas bidimensionais é baseada nos conceitos de "acurácia" obtidos em Baarda (1977) e em Monico et al. (2009). Neste último trabalho, a medida da acurácia de uma grandeza qualquer é dada por (MONICO et al., 2009):

$$
a=b \pm \sigma,
$$

onde na equação (9), $a$ é a medida de acurácia, $b$ é a (possível) tendência existente no valor estimado da grandeza, e $\sigma$ representa a sua precisão (incerteza). Nota-se que o conceito de acurácia envolve tanto a influência de erros não aleatórios (isto é, sistemáticos e/ou grosseiros), quanto os efeitos dos erros aleatórios. Na ausência de tendência (isto é, presença somente de erros aleatórios nas observações), o conceito de acurácia se confunde com o conceito de precisão (para maiores detalhes, ver MONICO et al., 2009). Esta definição para a acurácia de uma grandeza também está de acordo o conceito de "acurácia de uma rede geodésica" proposto em Baarda (1977), onde o referido autor afirma que a acurácia de uma rede geodésica consiste de duas partes: "precisão da rede geodésica" (relacionada a erros aleatórios) e "confiabilidade da rede geodésica" (relacionada à possível influência de erros não aleatórios não detectados). Para maiores detalhes, ver Baarda (1977) e também Klein et al. (2012).

Desta forma, na metodologia aqui proposta, para a estimativa da medida de tendência (b) na equação (9), será utilizada a análise de robustez, enquanto para a medida de precisão $(\sigma)$, será utilizada a análise de covariância tradicional.

Após estimar os elementos da matriz de deformação $\left(\mathrm{E}_{\mathrm{i}}\right)$, é possível estimar valores limites para as primitivas de tensão (parâmetros de robustez) em cada ponto $\mathrm{P}_{\mathrm{i}}$, considerando o deslocamento máximo "admissível" (tolerado) para as coordenadas deste ponto (ver BERBER et al., 2003). Entretanto, ao invés de se estimar valores limites para os parâmetros de robustez, pode-se retornar do campo de tensões (strain field) para o campo de deslocamento (displament field), e verificar se os deslocamentos dos pontos são de fato inferiores aos valores máximos admissíveis (tolerados). No caso bidimensional, estes deslocamentos nas coordenadas de um ponto $\mathrm{P}_{\mathrm{i}}$ (retornando do campo de tensões para o campo de deslocamento) são dados por (BERBER, 2006): 


$$
\left[\begin{array}{l}
\Delta x \\
\Delta y
\end{array}\right]=\left[\begin{array}{cc}
\frac{\partial \Delta x_{i}}{\partial x} & \frac{\partial \Delta x_{i}}{\partial y} \\
\frac{\partial \Delta y_{i}}{\partial x} & \frac{\partial \Delta y_{i}}{\partial y}
\end{array}\right]\left[\begin{array}{l}
x_{i}-x_{0} \\
y_{i}-y_{0}
\end{array}\right]
$$

onde na equação (10), $\Delta x$ e $\Delta y$ são, respectivamente, os deslocamentos nas coordenadas $\mathrm{x}_{\mathrm{i}}$ e $\mathrm{y}_{\mathrm{i}}$ do ponto $\mathrm{P}_{\mathrm{i}}$ (estimadas pelo ajustamento da rede), e $\mathrm{x}_{0}$ e $\mathrm{y}_{0}$ são as chamadas "condições iniciais" (initial conditions), sendo coordenadas que necessitam ser previamente calculadas, conforme será discutido a seguir.

A equação (10) é um sistema de equações diferenciais de primeira ordem, devendo ser solucionado por meio da sua integração numérica e determinação dos valores de contorno (ver, por exemplo, VANÍČEK e KRAKIWSKY, 1986). Em Berber (2006), entretanto, outra estratégia é adotada: minimizar a norma dos vetores de deslocamento de todos os pontos da rede geodésica, o que resultará em um deslocamento nulo para o centróide da mesma (sendo esta a estratégia adotada na metodologia aqui proposta). Por serem muito extensas, as fórmulas para a determinação das condições iniciais $\left(\mathrm{x}_{0}, \mathrm{y}_{0}\right)$ não serão aqui apresentadas, mas podem ser obtidas em Berber (2006, p. 90-92).

Após a determinação dos deslocamentos nas coordenadas de um ponto $\mathrm{P}_{\mathrm{i}}$ por meio da equação (10), a possível "tendência" (b), ou seja, o possível deslocamento total deste ponto no plano, devido a (possíveis) erros não detectados em uma observação, é dado por:

$$
b=\sqrt{\Delta x^{2}+\Delta y^{2}}
$$

Importante ressaltar que a equação (11), por ser a norma resultante do vetor de deslocamento $(\Delta \mathrm{x}, \Delta \mathrm{y})$ do ponto $\mathrm{P}_{\mathrm{i}}$, é uma medida datum independente. No caso, calcula-se o deslocamento total do ponto com a equação (11) para cada uma das $n$ matrizes de tensões para este (sendo cada uma relativa ao MDB de uma observação), e considera-se então somente o valor máximo obtido $-b_{\text {máx }}$.

Finalmente, para a estimativa da medida de precisão $(\sigma)$ na equação (9), propõe-se aqui o uso do semi-eixo maior da elipse de erros, obtida por meio da propagação das variâncias e covariâncias das observações sobre os parâmetros. Considerando que um ponto $\mathrm{P}_{\mathrm{i}}$ tenha suas coordenadas $\left(\mathrm{x}_{\mathrm{i}}, \mathrm{y}_{\mathrm{i}}\right)$ estimadas pelo ajustamento por mínimos quadrados da rede geodésica, a matriz de covariância de suas coordenadas, obtida pela lei de propagação de variâncias e covariâncias (ver, por exemplo, BAARDA, 1977; VANÍČEK e KRAKIWSKY, 1986, GHILANI e WOLF, 2006), é dada por: 


$$
\Sigma_{P_{i}}=\left[\begin{array}{cc}
\sigma_{x}^{2} & \sigma_{x y} \\
\sigma_{y x} & \sigma_{y}^{2}
\end{array}\right],
$$

onde na equação (12), $\sigma_{\mathrm{x}}{ }^{2}$ e $\sigma_{\mathrm{y}}{ }^{2}$ são as variâncias das coordenadas estimadas $\mathrm{x}_{\mathrm{i}}$ e $\mathrm{y}_{\mathrm{i}}$, respectivamente, e $\sigma_{\mathrm{xy}}=\sigma_{\mathrm{yx}}$ é a covariância entre estas. Com estes elementos, o semi-eixo maior da elipse de erros do ponto $P_{i}$ é dado por (SEEMKOOEI, 2001b):

$$
a_{P_{i}}=\sqrt{\frac{1}{2}\left(\sigma_{x}^{2}+\sigma_{y}^{2}+\sqrt{\left(\sigma_{x}^{2}-\sigma_{y}^{2}\right)+4 \sigma_{x y}^{2}}\right)}
$$

O semi-eixo maior da elipse de erros em um ponto $\mathrm{P}_{\mathrm{i}}$, define a incerteza (ou dispersão) "esperada" para este ponto no plano, segundo um determinado "grau de confiança", e o número de graus de liberdade do ajustamento, em função dos quais o valor do semi-eixo maior obtido em (13) deve ser escalonado (ver, por exemplo, GHILANI e WOLF, 2006). Portanto, a precisão, ou seja, o deslocamento máximo "esperado" para este ponto, devido à presença de erros aleatórios nas observações (de acordo com certo grau de confiança), é dado por:

$$
\sigma= \pm c a_{P_{i}}
$$

onde $c$ é o valor segundo o qual o semi-eixo maior da elipse de erros em (13) deve ser escalonado, obtido em função do grau de confiança estipulado e do número de graus de liberdade do ajustamento. Desta forma, na metodologia aqui proposta, a análise da acurácia deve ser feita para cada ponto $\left(\mathrm{P}_{\mathrm{i}}\right)$ da rede, analisando o deslocamento resultante do ponto (ver a Figura 2), e não as suas coordenadas separadamente, considerando tanto a influência de (possíveis) erros não detectados nas observações (via análise de robustez), quanto a influência dos (inevitáveis) erros aleatórios (via análise de covariância tradicional), com a vantagem dos resultados obtidos serem independente de fatores externos como o referencial utilizado, sendo reflexos da própria geometria/configuração da rede geodésica e do tipo e qualidade (propriedades estatísticas) de suas observações.

Salienta-se que estas discussões a respeito da independência do datum são relativas aos cenários cujo(s) ponto(s) de controle já estão previamente definido(s). Naturalmente, alterando a localização (e o número) de pontos de controle na rede, alteram-se os valores de acurácia para os demais pontos.

Finalmente, também é necessária uma atenção especial aos valores dos níveis de probabilidade assumidos $(\alpha, \gamma)$, dos quais se obtém o valor do parâmetro de não centralidade do modelo $\left(\lambda_{0}\right)$ em (4) (ver, por exemplo, VANÍČEK et al., 1996; KRAKIWSKY et al., 1999). Para demonstrar na prática a aplicação da metodologia proposta, um exemplo numérico é apresentado e discutido na próxima seção. 
Figura 2 - Ilustração gráfica das grandezas envolvidas na medida de acurácia posicional proposta para cada ponto (Fonte: Adaptado de Vaníček et al., 1996).

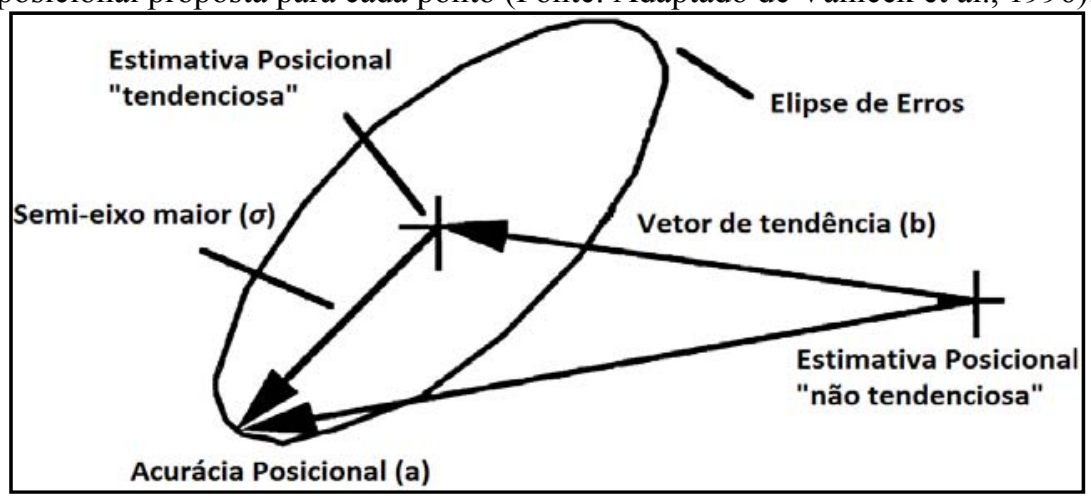

\section{EXEMPLO NUMÉRICO}

A rede geodésica utilizada neste exemplo demonstrativo é uma poligonal topográfica simulada que consiste de sete vértices, conforme ilustra a Figura 3.

Figura 3 - Poligonal topográfica no plano topográfico local simulado.

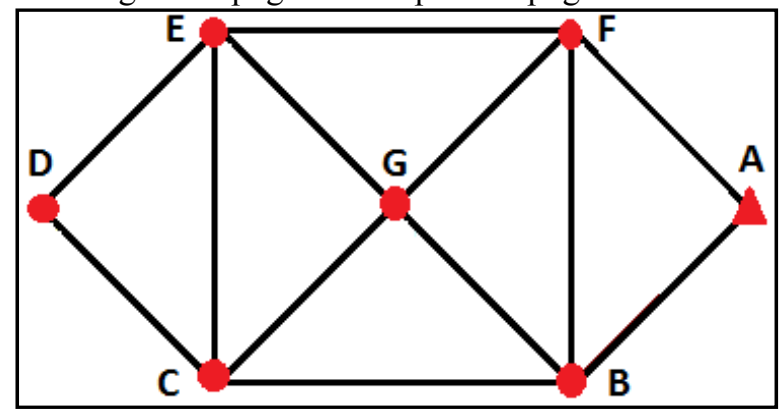

Analisando a Figura 3, nota-se que a rede geodésica horizontal em estudo consiste de seis vértices desconhecidos (B, C, D, E, F e G), e um ponto de controle (A). As observações simuladas foram as distâncias entre os pares de vértices interligados na Figura 3, bem como os ângulos internos entre os pontos BAF, FGB, BGC, CGE, EGF e EDC. As injunções (absolutas) consideradas foram as coordenadas do vértice $A$ no plano topográfico local simulado $\left(\mathrm{X}_{\mathrm{A}}=10.000 \mathrm{~m}, \mathrm{Y}_{\mathrm{A}}=\right.$ $10.000 \mathrm{~m})$, e o azimute do alinhamento $\mathrm{AB}\left(\mathrm{Az}_{\mathrm{AB}}=225^{\circ}\right)$, ou seja, foi considerado um sistema minimamente injuncionado. Desta forma, o ajustamento consiste de 18 observações (doze medidas de distâncias e seis medidas de ângulos) e 12 parâmetros (coordenadas planimétricas $\mathrm{X}, \mathrm{Y}$ dos seis vértices desconhecidos), sendo realizado 
com seis graus de liberdade. Embora neste caso sejam utilizadas somente injunções absolutas, o método também é aplicável utilizando injunções relativas.

Neste exemplo, o valor dos ângulos internos considerados, "isentos" de erros, é $90^{\circ}$ (com uma precisão estipulada de 2"), o valor das distâncias (horizontais e verticais) "isentas" de erros é $1000 \mathrm{~m}$ (com uma precisão estipulada de $6 \mathrm{~mm}$ ), e o valor das distâncias nas demais direções do plano topográfico local, "isentas" de erros, é 707,107 m (com uma precisão estipulada de $5 \mathrm{~mm}$ ). Desta forma, considerando as precisões estipuladas, foram numericamente gerados e inseridos erros aleatórios nestes valores "isentos" de erros das observações, e com estes "novos valores" para as observações (considerando os erros aleatórios numericamente gerados e inseridos), foi realizado o ajustamento por mínimos quadrados da rede, resultando nas matrizes e vetores utilizados posteriormente para a etapa de controle de qualidade. No caso da matriz peso $(\mathrm{P})$, esta foi tomada como sendo a inversa da matriz de covariância das observações.

Para o cálculo dos parâmetros de robustez da rede, inicialmente foram calculadas as medidas de confiabilidade interna e externa das observações, por meio das equações (4) e (5), considerando um nível de significância de $\alpha=5 \%$ e um poder do teste de $\gamma=95 \%$, o que resulta em um valor para o parâmetro de não centralidade do modelo de $\lambda_{0}=13,0321$ (ver, por exemplo, BAARDA, 1968; VANÍČEK et al., 1991; TEUNISSEN, 2006). Com estas medidas de confiabilidade, utilizando a equação (8), se calculou os parâmetros de robustez de todos os pontos desconhecidos da rede (considerando separadamente a confiabilidade externa gerada por cada observação), sendo os valores máximos obtidos para as primitivas de tensão (parâmetros de robustez $-\sigma_{\text {máx }}, \omega_{\text {máx }}, \gamma_{\text {máx }}$ ) apresentados, em módulo, na Tabela 1 (com $\sigma_{\text {máx }}$ e $\gamma_{\text {máx }}$ em ppm - partes por milhão, e $\omega_{\operatorname{máx}}$ em $\left.\mu \mathrm{rad}\right)$. Ressalva-se que para o cálculo dos elementos das matrizes de tensões em (7), se considerou somente os vértices desconhecidos interligados ao ponto de interesse por pelo menos uma observação (ver, por exemplo, VANÍČEK et al., 1991; VANÍČEK et al., 1996).

Tabela 1 - Parâmetros de robustez para os vértices desconhecidos da rede.

\begin{tabular}{c|c|c|c|c|c|c}
\hline $\begin{array}{c}\text { Parâmetro de } \\
\text { robustez }\end{array}$ & \multicolumn{6}{|c}{ Vértice desconhecido } \\
\cline { 2 - 7 } & $\mathbf{B}$ & $\mathbf{C}$ & $\mathbf{D}$ & $\mathbf{E}$ & $\mathbf{F}$ & $\mathbf{G}$ \\
\hline $\boldsymbol{\sigma}_{\text {máx }}(\mathbf{e m} \mathbf{p p m})$ & 9,63 & 11,1 & 22,6 & 14,0 & 12,0 & 6,77 \\
\hline $\boldsymbol{\omega}_{\text {máx }}(\mathbf{e m} \boldsymbol{\mu} \mathbf{r a d})$ & 25,4 & 23,5 & 24,7 & 18,8 & 19,6 & 21,9 \\
\hline$\gamma_{\text {máx }}(\mathbf{e m} \mathbf{p p m})$ & 17,0 & 12,3 & 38,7 & 16,2 & 23,5 & 16,8 \\
\hline
\end{tabular}

Analisando a Tabela 1, nota-se que o ponto desconhecido que apresenta a maior deficiência em escala e em configuração local é o vértice D. Estes resultados não são surpreendentes, uma vez que o vértice $\mathrm{D}$ é o ponto desconhecido mais afastado do ponto de controle (A), além de estar relacionado com somente três observações (duas distâncias e um ângulo), ao contrário do vértice G, por exemplo, 
relacionado com oito observações (4 medidas de distâncias e 4 ângulos). Além disso, os vértices B e D são os únicos relacionados com somente uma observação angular, e, desta forma, são os mais deficientes em orientação (embora neste caso, os valores dos parâmetros de robustez da rotação diferencial média dos vértices estejam mais próximos do que para a dilatação média e o cisalhamento total). Interessante notar também que, embora seja o vértice com mais observações redundantes na rede, o ponto $\mathrm{G}$ é o mais robusto em escala, mas não é o mais robusto em rotação e em configuração local (sendo estes os vértices $\mathrm{E}$ e C, respectivamente).

Estes resultados demonstram como os parâmetros de robustez fornecem uma análise do potencial de deformação da rede diferente da obtida por meio da confiabilidade externa, salientando que estes parâmetros são ainda independentes do referencial (datum) utilizado. Caso desejado, pode-se definir valores limites para os parâmetros de robustez, em virtude dos deslocamentos máximos "tolerados" para os vértices da rede, tanto na etapa de pré-análise (planejamento), quanto na etapa de controle de qualidade de uma rede já existente (ver BERBER et al., 2003).

Para a aplicação da metodologia de controle de qualidade aqui proposta, primeiro, é necessário retornar do campo de tensões para o campo de deslocamentos, para estimar os deslocamentos máximos dos pontos via análise de robustez (estimativa de tendência), e ainda, o semi-eixo maior da elipse de erros em cada ponto (para complementar a estimativa de acurácia posicional destes). Desta forma, considerando a confiabilidade externa gerada por cada observação, foram calculadas as condições iniciais da rede $\left(\mathrm{x}_{0}, \mathrm{y}_{0}\right)$, e com estas, se determinou os deslocamentos dos pontos, por meio da equação (10). Após isto, por meio da equação (11), se determinou o deslocamento resultante de cada ponto no plano topográfico local (considerando a confiabilidade externa gerada por cada observação separadamente), sendo que os valores máximos para os deslocamentos dos pontos, obtidos desta forma, são apresentados na Tabela 2.

Tabela 2 - Deslocamento máximo para os vértices desconhecidos da rede.

\begin{tabular}{c|c|c|c|c|c|c}
\hline & \multicolumn{6}{|c}{ Vértice desconhecido } \\
\cline { 2 - 7 } & B & C & D & E & F & G \\
\hline $\begin{array}{c}\text { Deslocamento } \\
\text { máximo resultante - } \\
\mathbf{b}_{\text {máx }}(\mathbf{m})\end{array}$ & 0,022 & 0,014 & 0,021 & 0,014 & 0,020 & 0,007 \\
\hline
\end{tabular}

Analisando a Tabela 2, nota-se que no campo de deslocamentos, o vértice $\mathrm{G}$ é o menos afetado por possíveis erros não detectados nas observações, enquanto os vértices $B, D$ e $F$ são os mais afetados, logo, as conclusões são similares as que foram obtidas na análise realizada no campo de tensões (ou seja, por meio dos parâmetros de robustez de cada vértice). 
Seguindo a metodologia proposta, resta ainda calcular o semi-eixo maior da elipse de erros dos vértices desconhecidos da rede. Salienta-se que esta abordagem é realizada por meio da análise de covariância tradicional, e está relacionada com a influência de erros aleatórios, ao contrário da análise de robustez, relacionada com a influência de possíveis erros não aleatórios (não detectados). De qualquer forma, o grau de confiança para as elipses de erros foi o mesmo utilizado para o cálculo das medidas de confiabilidade (ou seja, de $1-\alpha=0,95$ ). Para este grau de confiança, considerando os seis graus de liberdade do ajustamento, os valores calculados para o semi-eixo maior das elipses por meio da equação (13) devem ser multiplicados pela constante $\mathrm{c}=3,21$ (para maiores detalhes, ver, por exemplo, GHILANI e WOLF, 2006), sendo os resultados obtidos apresentados na Tabela 3.

Tabela 3 - Valores para o semi-eixo maior da elipse de erros dos pontos.

\begin{tabular}{c|c|c|c|c|c|c}
\cline { 2 - 7 } & \multicolumn{6}{c}{ Vértice desconhecido } \\
\cline { 2 - 7 } & B & $\mathbf{C}$ & $\mathbf{D}$ & $\mathbf{E}$ & $\mathbf{F}$ & $\mathbf{G}$ \\
\hline Semi-eixo maior $-\boldsymbol{\sigma}(\mathbf{m})$ & 0,022 & 0,059 & 0,077 & 0,061 & 0,029 & 0,037 \\
\hline
\end{tabular}

Analisando a Tabela 3, nota-se que o tamanho da elipse de erros aumenta conforme o afastamento do ponto desconhecido em relação ao ponto de controle da rede (ver também a Figura 3), ou seja, de fato a análise de robustez (relacionada a possíveis "erros não detectados") e a análise de covariância (relacionada à propagação de "erros aleatórios") fornecem resultados diferentes e complementares do potencial de deformação de uma rede geodésica, conforme já apontado, por exemplo, em Vaníček et al. (2001). Desta forma, a Tabela 4 apresenta a acurácia posicional dos vértices de acordo com a metodologia proposta, considerando o caso extremo em que o semi-eixo maior da elipse de erros $(\sigma)$ e o deslocamento máximo resultante devido a erros não detectados em uma dada observação $\left(b_{\text {máx }}\right)$ são paralelos e possuem o mesmo sentido (ver a Figura 2), considerando ainda um mesmo grau de confiança tanto para os valores obtidos por meio da análise de robustez quanto para os valores obtidos por meio da análise de covariância.

Tabela 4 - Acurácia posicional dos vértices de acordo com a metodologia proposta.

\begin{tabular}{c|c|c|c|c|c|c}
\hline Grau de confiança para a & \multicolumn{7}{|c}{ Vértice desconhecido } \\
\cline { 2 - 7 } acurácia posicional $=\mathbf{9 5 \%}$ & $\mathbf{B}$ & $\mathbf{C}$ & $\mathbf{D}$ & $\mathbf{E}$ & $\mathbf{F}$ & $\mathbf{G}$ \\
\hline Vetor de tendência $-\mathbf{b}_{\text {máx }}(\mathbf{m})$ & 0,022 & 0,014 & 0,021 & 0,014 & 0,020 & 0,007 \\
\hline Vetor de incerteza $-\boldsymbol{\sigma}(\mathbf{m})$ & 0,022 & 0,059 & 0,077 & 0,061 & 0,029 & 0,037 \\
\hline Acurácia posicional $-\mathbf{a}(\mathbf{m})$ & 0,044 & 0,073 & 0,098 & 0,075 & 0,049 & 0,044 \\
\hline
\end{tabular}

Analisando a Tabela 4, nota-se que de fato os resultados obtidos por meio da análise de robustez são diferentes dos resultados obtidos por meio da análise de covariância, devendo ser analisados de maneira complementar. Neste exemplo, a influência de erros aleatórios, especialmente para os pontos mais afastados do ponto 
de controle da rede, é maior do que a influência de possíveis erros não detectados nas observações, considerando um grau de confiança de 95\% para ambas as abordagens. De acordo com a metodologia proposta, se a acurácia posicional estipulada para os vértices desconhecidos da rede fosse de $10 \mathrm{~cm}$ (com 95\% de confiança), todos os vértices estariam dentro deste limite tolerável, mas, se a acurácia posicional estipulada fosse, por exemplo, de $5 \mathrm{~cm}$, somente os vértices $\mathrm{B}, \mathrm{F}$ e G estariam dentro deste limite tolerável, e a rede geodésica, portanto, deveria ser "melhorada" (com a inclusão de novas observações/vértices/pontos de controle, e/ou com a repetição das observações, porém com equipamentos e métodos mais precisos, especialmente para a região "mais fraca" da rede, em torno dos pontos C, $\mathrm{D}$ e E). Desta forma, este exemplo demonstra como a metodologia proposta pode ser aplicada, por exemplo, de acordo com as normas vigentes para o georreferenciamento de imóveis rurais (ver INCRA, 2010), para o cadastro técnico urbano (quando vigente no país), ou para qualquer outra norma que estipule critérios de acurácia para redes geodésicas materializadas por vértices.

\section{CONCLUSÃO}

Neste trabalho se apresentou uma proposta metodológica para o controle de qualidade de redes geodésicas horizontais. Na metodologia proposta, a acurácia (exatidão) posicional de cada ponto em uma rede é estimada por meio de uma possível tendência em sua posição (via análise de robustez), além de sua própria precisão (incerteza) posicional (via análise de covariância), com a vantagem dos resultados obtidos serem independentes do referencial (datum) adotado.

Para mostrar a aplicação da metodologia apresentada, um exemplo numérico de caráter ilustrativo foi apresentado, envolvendo uma poligonal topográfica numericamente simulada. Neste exemplo demonstrativo, os resultados obtidos foram concordantes com as premissas teóricas, pois, em geral, quanto maior o afastamento de um vértice desconhecido do(s) ponto(s) de controle da rede, maior é a propagação de erros aleatórios sobre este, e, quanto menos observações redundantes ao redor de um ponto, maior é a influência de possíveis erros (não aleatórios) sobre este, quando não detectados. Desta forma, a acurácia posicional de cada vértice da rede deve considerar os resultados obtidos por meio das duas abordagens, conforme fora aqui apresentado.

Este estudo inicial tem como principal objetivo apresentar o método proposto, que no momento se limita a redes bidimensionais. Como sugestões para trabalhos futuros, podem-se citar investigações sobre o planejamento (ou pré-análise) de redes geodésicas, seguindo a metodologia apresentada, a questão de redes geodésicas com injunções relativas, como selecionar de maneira adequada os níveis de probabilidade assumidos para as medidas de confiabilidade e para as elipses de erros (considerando tanto os custos envolvidos quanto a acurácia desejada), a adaptação da metodologia proposta para uma determinada norma técnica vigente, além de sua aplicação em redes geodésicas unidimensionais e tridimensionais. 


\section{AGRADECIMENTOS:}

Os autores agradecem à FAPERGS/CAPES pelo fornecimento da bolsa de Doutorado do primeiro autor, ao CNPq pela Bolsa de Produtividade em Pesquisa (Proc.n.303306/2012-2) do segundo autor e do terceiro autor (Proc.n.305041/20061), e pelo projeto Universal (proc. n. 477914/2012-8).

\section{REFERÊNCIAS BIBLIOGRÁFICAS}

BAARDA, W. A testing procedure for use in geodetic networks. Publications on Geodesy, New Series, v. 2, n. 5, Delft: Netherlands Geodetic Commission, 1968.

BAARDA, W. Measures for the accuracy of geodetic networks. In: Symposium on Optimization of Design and Computation of Control Networks, p. 419-436, Sopron, Hungary, 1977.

BERBER, M.; DARE, P. J.; VANÍČEK, P.; CRAYMER, M. R. On The Application Of Robustness Analysis To Geodetic Networks. In: Proceedings of Canadian Society for Civil Engineering, 31st Annual Conference, June 4-7, Moncton, NB, Canada, 2003.

BERBER, M.; DARE, P. J.; VANÍČEK, P. Robustness Analysis of TwoDimensional Networks. Journal of Surveying Engineering, v. 132, n. 4, p. 168174, 2006.

BERBER, M.; VANÍČEK, P.; DARE, P. J. Robustness Analysis of 3D Networks. Journal of Geodynamics, v. 47 n. 1, p. 1-8, 2009.

BERBER, M. Robustness Analysis of Geodetic Networks. Ph.D. Dissertation Department of Geodesy and Geomatics Engineering, University of New Brunswick, Fredericton, Canada, 2006. 121p.

CAVALHERI, E. P.; CHAVES, J.C. Análises Geométrica e de Confiabilidade na Robustez de Redes. In: Anais do III Simpósio Brasileiro de Geomática, 25-27 Julho, Presidente Prudente, São Paulo, Brasil, v. 1, p. 325-328, 2012a.

CAVAlHERI, E. P.; CHAVES, J.C. Determinação da Confiabilidade Interna e Externa no Processo de Robustez de Redes Geodésicas. In: Anais do IV Simpósio Brasileiro de Ciências Geodésicas e Tecnologia da Informação, 0609 Maio, Recife, Pernambuco, Brasil, 2012b.

CRAYMER, M. R.; VANÍČEK, P. Corrections to robustness analysis of geodetic horizontal networks. Journal of Geodesy, v. 76, n. 8, p. 476, 2002.

DARE, P. Strength Analysis of Horizontal Networks Using Strain. Survey Science Tech. Rep. 2, University of Toronto, Erindale Campus, Mississauga, Ontario, 1983.

GAMBIN, W.; PARZYÑSKI, Z.; PRÓSZYÑSKI W. The Concept of Network Robustness based on Strain Analogy as seen in the Light of Continuum Mechanics. In: Proceedings of FIG/IAG Symposium Measuring the Changes, May 12-15, Lisbon, Portugal, 2008.

GHILANI, C. D.; WOLF, P. R. Adjustment Computations: Spatial Data Analysis. 4. ed. New York: John Wiley \& Sons, 2006. 611p. 
HETNARSKI, R. B.; IGNACZAK, J. The Mathematical Theory of Elasticity, 2. ed. CRC Press, 2010. 800p.

HSU, R.; LI, S. Decomposition of deformation primitives of horizontal geodetic networks: Application to Taiwan's GPS network. Journal of Geodesy, v. 78, n. 4-5, p. 251-262, 2004.

HSU, R.; LEE, H. C.; KAO, S. P. Three-Dimensional Networks Are Horizontally Superior in Robustness: A Mathematical Reasoning. Journal of Surveying Engineering, v. 132, n. 2, p. 61-65, 2008.

INSTITUTO NACIONAL DE COLONIZAÇÃO E REFORMA AGRÁRIA (INCRA). Ministério de Desenvolvimento Agrário (MDA). Normas técnicas para georreferenciamento de imóveis rurais. 2. ed. Brasília, 2010.

KASAHARA, K. Physical conditions of Earthquake Faults as Deduced from Geodetic Observations. Bulletin of the Earthquake Research Institute. University of Tokyo, v. 36, p. 455-464, 1958.

KLEIN, I.; MATSUOKA, M. T.; SOUZA, S. F.; COLlisCHONN, C. Planejamento de Redes Geodésicas Resistentes a Múltiplos Outliers. Boletim de Ciências Geodésicas, v. 18, n. 3, p. 480-507, 2012.

KRAKIWSKY, E. J., D. J. SZABO, P. VANÍCEK, AND M. R. CRAYMER. Development and testing of in-context confidence regions for geodetic survey networks. Final contract report, Department of Geodesy and Geomatics Engineering Technical Report No. 198, University of New Brunswick, Fredericton, New Brunswick, Canada, 1999. 24 pp.

LOVE, A. E. H. A Treatise on the Mathematical Theory of Elasticity. Dover Publications, New York, 1944.

MARJETIČ, A.; AMBROŽIČ, T.; TURK, G.; STERLE, O.; STOPAR, B. Statistical properties of Strain and Rotation Tensors in Geodetic Network. Journal of Surveying Engineering, v. 136, n. 3, p. 102-110, 2010.

MONICO, J. F. G.; PÓZ, A. P. D.; GALO, M.; SANTOS, M. C.; OLIVEIRA, L. C. Acurácia e precisão: revendo os conceitos de forma acurada. Boletim de Ciências Geodésicas, v. 15, n. 3, p. 469-483, 2009.

ONG, P. J. Robustness Analysis for Geodetic Networks. Master of Science Thesis in Engineering, Department of Surveying Engineering, University of New Brunswick, Fredericton, Canada, 1993.

POPE, A. Strain Analysis of Repeated Triangulation for the Analysis of Crustal Movement. Master of Science Thesis, Department of Geodetic Science, The Ohio State University, Colombus, Ohio, USA, 1966.

SCHNEIDER, D. Complex crustal strain approximation. Department of Surveying Engineering Technical Report No. 91, University of New Brunswick, Fredericton, New Brunswick, 1982.

SEEMKOOEI, A. A. Comparison of reliability and geometrical strength criteria in geodetic networks. Journal of Geodesy, v. 75, n. 4, p. 227-233, 2001 a. 
SEEMKOOEI, A. A. Strategy for designing geodetic networks with high reliability and geometrical strength. Journal of Surveying Engineering, v. 127 n.3, p. 104-117, 2001b.

TEIXEIRA, N. N.; FERREIRA, L. D. D. Determinação e Análise Estatística dos Parâmetros Envolvidos no Monitoramento de Deformações. Boletim de Ciências Geodésicas, Curitiba, v. 8, n. 2, p. 21-35, 2002.

TERADA, T.; MIYABE, N. Deformation of the Earth Crust in Kwansai Districts and its Relation to the Orographic Feature. Bulletin of the Earthquake Research Institute. University of Tokyo, v. 7, Part 2, p. 223-241, 1929.

TEUNISSEN, P. J. G. Testing theory: an introduction. 2. ed. Delft: Ed. VSSD, 2006. 147p.

THAPA, K. Strain as a Diagnostic Tool to Identify Inconsistent Observations and Constrains in Horizontal Geodetic Networks. Department of Surveying Engineering, Technical Report No:68, UNB, Fredericton, Canada, 1980.

TSUBOI, C. Investigation on the deformation of the earth's crust in the Tango district connected with the Tango earthquake of 1927 (Part IV). Bulletin of the Earthquake Research Institute. University of Tokyo, v. 10, p. 411-434, 1932.

VANÍČEK, P.; CRAYMER, M. R.; KRAKIWSKY, E. J. Robustness analysis of geodetic horizontal networks. Journal of Geodesy, v. 75, n. 4, p. 199-209, 2001.

VANÍČEK, P.; GRAFAREND, E. W.; BERBER, M. Short Note: Strain invariants. Journal of Geodesy, v. 82, n. 4-5, p. 263-268, 2008.

VANÍČEK, P.; KRAKIWSKY, E. J.; CRAYMER, M. R.; GAO, Y.; ONG, P. J. Robustness analysis. Final contract report, Department of Surveying Engineering Technical Report No. 156, University of New Brunswick, Fredericton, New Brunswick, Canada, 1990. 116p.

VANÍČEK, P.; KRAKIWSKY, E. J. Geodesy: The concepts, 2. ed. North Holland/Elsevier, Amsterdam, 1986.

VANÍČEK, P.; ONG, P.J.; KRAKIWSKY, E.J.; CRAYMER, M.R. Application of Robustness analysis to Large Geodetic Networks. Final contract report, Department of Geodesy and Geomatics Engineering Technical Report No. 180, University of New Brunswick, New Brunswick, Canada, 1996. 75p.

VANÍČEK, P.; THAPA, K.; SCHNEIDER, D. The use of strain to identify incompatible observations and constraints in horizontal geodetic networks. Manuscripta Geodaetica, v. 6, p. 257-281, 1981.

(Recebido em maio de 2013. Aceito em julho de 2013). 